\title{
EXISTENCE OF STEADY WAVES FOR A CLASS OF NONLINEAR DISSIPATIVE MATERIALS*
}

\author{
BY \\ JAMES M. GREENBERG \\ Mellon Institute, Pittsburgh, Pennsylvania
}

1. Introduction. In recent years there has been extensive work on wave propagation in nonlinear dissipative materials. Coleman, Gurtin, and Herrera [1] have studied shock waves in materials obeying the fading memory hypothesis, while acceleration waves and higher order discontinuities in such materials have been investigated by Coleman and Gurtin [1]-[4], Varley [5], Coleman, Greenberg, and Gurtin [6], and Wang and Bowen [7]. Dunwoody [8] has studied acceleration waves in linear fluent bodies.

Pipkin [9], using a special constitutive equation of the fading memory type, obtained exact solutions to the one-dimensional steady flow equations which possessed both acceleration and shock waves. Generalizing a portion of Pipkin's work [10], I showed that for a large class of nonlinear viscoelastic materials satisfying the fading memory hypothesis it is possible to find one-dimensional steady solutions exhibiting shock waves.

In this present paper I assume that the stress $\sigma$ is related to the deformation gradient $F$ through the following constitutive equation:

$$
\sigma_{t}=E(F, \sigma) F_{t}+G(F, \sigma) .
$$

I show that if $E$ and $G$ satisfy certain hypotheses, there exist steady solutions of the one-dimensional flow equations which possess both shock and acceleration waves.

2. Steady motions and governing balance laws. In what follows $R$ will be the real line. Material points in $R$ will be denoted by $X$ and the parameter $t$ in $R$ will represent time. A function $\chi$ will be called a motion if it is continuous and has partial derivatives $\chi_{x}>0$ and $\chi_{t}$ which are piecewise continuous on $R \times R$. The spacial derivative $\chi_{x}$ is called the deformation gradient and the time derivative $\chi_{t}$ the velocity.

A motion $\chi$ is said to be steady if there exist positive-valued, piecewise continuous functions $\hat{F}$ and $\hat{u}$ such that

$$
\chi_{X}(X, t)=\hat{F}(x) \text { and } \chi_{t}(X, t)=\hat{u}(x),
$$

where $x=\chi(X, t)$ is the place occupied by the material point $X$ at time $t$. It is not difficult to show that $\chi$ is steady if and only if there exists a positive number $V_{0}$ and a continuous function $f$ having a piecewise continuous derivative $f^{\prime}>0$ such that

$$
\chi(X, t)=f(\xi), \quad \xi=X / V_{0}+t
$$

for all $X$ and $t$. For steady motions such that $\lim _{\xi \rightarrow-\infty} f^{\prime}(\xi)=V_{0}$, the parameter $V_{0}$ may be interpreted as the upstream velocity, that is the velocity at the point in space $x=-\infty$.

*Received January 19, 1967.

${ }^{1}$ Noll [11] discusses materials for which (1.1) holds. The constitutive equation used by Dunwoody [8] is similar to (1.1) and that used by Pipkin [9] is a special case of (1.1).

${ }^{2} \mathrm{Cf}$. [10, Thm. 2.1]. 
We say that a stress field $\sigma$ is steady if there exists a function $\hat{\sigma}$ such that

$$
\sigma(X, t)=\hat{\sigma}(x)
$$

where again $x=\chi(X, t)$. If the stress field and the motion are both steady, then clearly the stress $\sigma$ is a function of the variable $\xi=X / V_{0}+t$.

If we assume that the density of material points $X$ in $R$ is a constant $\rho_{R}$, then in the absence of body forces the stress $\sigma$ and the motion $\chi$ are related through balance of momentum:

$$
\frac{d}{d t} \int_{X_{1}}^{X_{2}} \rho_{R} \chi_{t}(X, t) d X=\sigma\left(X_{2}, t\right)-\sigma\left(X_{1}, t\right)
$$

for all $X_{1}, X_{2}$, and $t$. Since $\partial / \partial X=\left(1 / V_{0}\right)(d / d \xi)$ and $\partial / \partial t=d / d \xi$ it is not difficult to show that for steady motions and steady stress fields (2.4) reduces to

$$
\sigma\left(\xi_{2}\right)-\sigma\left(\xi_{1}\right)-\mu\left(F\left(\xi_{2}\right)-F\left(\xi_{1}\right)\right)=0
$$

for all $\xi_{1}$ and $\xi_{2}$ in $R$ where

$$
\begin{aligned}
\chi(X, t) & =f(\xi), \quad \xi=X / V_{0}+t, \\
\chi_{X}(X, t) & =\left(1 / V_{0}\right) f^{\prime}(\xi) \stackrel{\text { def }}{=} F(\xi), \\
\chi_{t}(X, t) & =f^{\prime}(\xi), \quad \text { and } \quad \mu=\rho_{R} V_{0}^{2} .
\end{aligned}
$$

We note that if $\lim _{\xi \rightarrow-\infty} f^{\prime}(\xi)=V_{0}$, then $\lim _{\xi \rightarrow-\infty} F(\xi)=1$.

For steady motions and stress fields (1.1) becomes

$$
d \sigma / d \xi=E(F, \sigma)(d F / d \xi)+G(F, \sigma) .
$$

Before discussing the solutions of the system of Eqs. (2.5) and (2.7) we make

Assumption 2.1. (a) The functions $E$ and $G$ are, respectively, $C^{1}\{(0, \infty) \times$ $(-\infty, \infty)\}$ and $C^{2}\{(0, \infty) \times(-\infty, \infty)\}$ and in $(0,1] \times(-\infty, 0]$ they satisfy:

$$
\begin{gathered}
E>0, \quad E_{F}<0, \quad \text { and } E_{\sigma}<0, \\
G_{F}>0 \text { and } G_{\sigma}<0 .
\end{gathered}
$$

(b) There exists a unique, $C^{2}$ function $\sigma_{E}$ on $(0,1]$ with the following properties:

$$
\begin{gathered}
\sigma_{E}(1)=0 \\
G\left(F, \sigma_{E}(F)\right)=0, \\
\sigma_{E}^{\prime}(F) \stackrel{\text { def }}{=} \frac{d \sigma_{E}(F)}{d F}=-\frac{G_{F}\left(F, \sigma_{E}(F)\right)}{G_{\sigma}\left(F, \sigma_{E}(F)\right)}<E\left(F, \sigma_{E}(F)\right), \\
\sigma_{E}^{\prime \prime}(F) \stackrel{\text { def }}{=} \frac{d^{2} \sigma_{E}(F)}{d F^{2}}<0, \\
\lim _{F \rightarrow 0^{+}} \sigma_{E}^{\prime}\left(F^{\prime}\right)=\infty, \text { and } \\
\lim _{F \rightarrow 0^{+}} \sigma_{E}^{\prime \prime}(F)=-\infty .
\end{gathered}
$$

Remark 2.1. It is possible to show that if $E$ and $G$ satisfy Assumption 2.1, then IIt is a consequence of $(2.8 \mathrm{~b})$ that $\sigma_{E}^{\prime}>0$ in $(0,1]$. 
materials governed by the constitutive Eq. (1.1) exhibit both stress relaxation and creep effects.

3. The existence of compressive loading steady flows which exhibit shock and acceleration waves. In the sequel we shall assume that Assumption 2.1 holds. Steady fields $(F, \sigma)$ which satisfy (2.5) and (2.7) are now sought. We say that a pair of functions $(F, \sigma)$ are compressive loading if they are bounded and monotone decreasing in $R$ and satisfy the upstream condition

$$
\lim _{\xi \rightarrow-\infty} F(\xi)=1 \text { and } \lim _{\xi \rightarrow-\infty} \sigma(\xi)=\sigma_{E}(1)=0 .
$$

By a compressive loading solution we mean a pair of functions $(F, \sigma)$ which are compressive loading, obey (2.5) everywhere, and obey (2.7) almost everywhere in $R$. We note that the pair $(F, \sigma) \equiv(1,0)$ is trivially a compressive loading solution. In the sequel this pair is called the trivial solution.

REMARK 3.1. A pair of compressive loading functions $(F, \sigma)$ define a compressive loading solution if and only if they satisfy

and

$$
\sigma=\mu(F-1), \quad \text { for all } \xi \in R
$$

$$
d \sigma / d \xi=E(F, \sigma)(d F / d \xi)+G(F, \sigma),
$$

almost everywhere in $R$.

Proof. Necessity follows from (2.5), (2.7), and (3.1); sufficiency is immediately evident.

REMARK 3.2. There exists a unique, $C^{2}$ function $\sigma_{I}$ which satisfies the initial value problem:

$$
\begin{gathered}
\frac{d \sigma_{I}}{d F}=E\left(F, \sigma_{I}\right), \quad F \in(0,1), \\
\sigma_{I}(1)=\sigma_{E}(1)=0 .
\end{gathered}
$$

Moreover, $\sigma_{I}$ has the following additional properties:

$$
\begin{gathered}
\sigma_{I}(F)<\sigma_{E}(F), \quad F \in(0,1), \\
E\left(F, \sigma_{I}(F)\right)>\sigma_{E}^{\prime}(F), \quad F \in(0,1],
\end{gathered}
$$

and

$$
d^{2} \sigma_{I}(F) / d F^{2}=E_{F}\left(F, \sigma_{I}(F)\right)+E_{\sigma}\left(F, \sigma_{I}(F)\right) E\left(F, \sigma_{I}(F)\right)<0, \quad F \in(0,1] .
$$

Proof. The existence and uniqueness of a function $\sigma_{I}$ satisfying (3.4) follows from Assumption 2.1 (a) and from classical theorems on the existence and uniqueness of solutions of ordinary differential equations.

Equations (3.5)-(3.7) follow immediately from equations (2.8a) and (2.9c).

For compressive loading functions we define the upstream equilibrium sound speed $V_{E}$ and the upstream instantaneous sound speed $V_{I}$ as follows:

$$
\begin{aligned}
V_{E} & =\left(\left.\frac{1}{\rho_{R}} \frac{d \sigma_{E}}{d F}\right|_{F=1}\right)^{1 / 2}=\left(\frac{-1}{\rho_{R}} \frac{G_{F}(1,0)}{G_{\sigma}(1,0)}\right)^{1 / 2}, \\
V_{I} & =\left(\frac{1}{\rho_{R}} E(1,0)\right)^{1 / 2} .
\end{aligned}
$$

${ }^{4}(3.1)$ is merely a statement that at $-\infty$ an equilibrium situation exists. 
Since $\mu=\rho_{R} V_{0}^{2}$, one has

$$
\begin{aligned}
V_{0} & \leq V_{E} \Leftrightarrow \mu \leq \sigma_{E}^{\prime}(1), \\
V_{E}<V_{0} & <V_{I} \Leftrightarrow \sigma_{E}^{\prime}(1)<\mu<E(1,0), \\
\text { and } \quad V_{I} & \leq V_{0} \Leftrightarrow E(1,0) \leq \mu .
\end{aligned}
$$

REMARK 3.3. (i) If $V_{0}>V_{E}$, there exists a unique number $F_{\infty} \in(0,1)$ which satisfies

$$
\sigma_{E}\left(F_{\infty}\right)=\mu\left(F_{\infty}-1\right)
$$

where $\sigma_{E}$ is defined in Assumption 2.1 (b). Moreover,

$$
\sigma_{E}^{\prime}\left(F_{\infty}\right)-\mu>0 .
$$

(ii) If $V_{0}>V_{I}$, there exists a unique number $F_{0} \in(0,1)$ which satisfies

$$
\sigma_{I}\left(F_{0}\right)=\mu\left(F_{0}-1\right),
$$

where $\sigma_{I}$ is defined in Remark 3.2. In addition,

$$
\sigma_{I}^{\prime}\left(F_{0}\right)-\mu>0
$$

(iii) If the hypotheses of (ii) hold, then $F_{\infty}$ and $F_{0}$ are ordered in the following way:

$$
0<F_{\infty}<F_{0}<1 \text {. }
$$

For a pictorial representation of the implications of Assumption 2.1(b) and Remarks 3.2 and 3.3, the reader is referred to Figure 1.

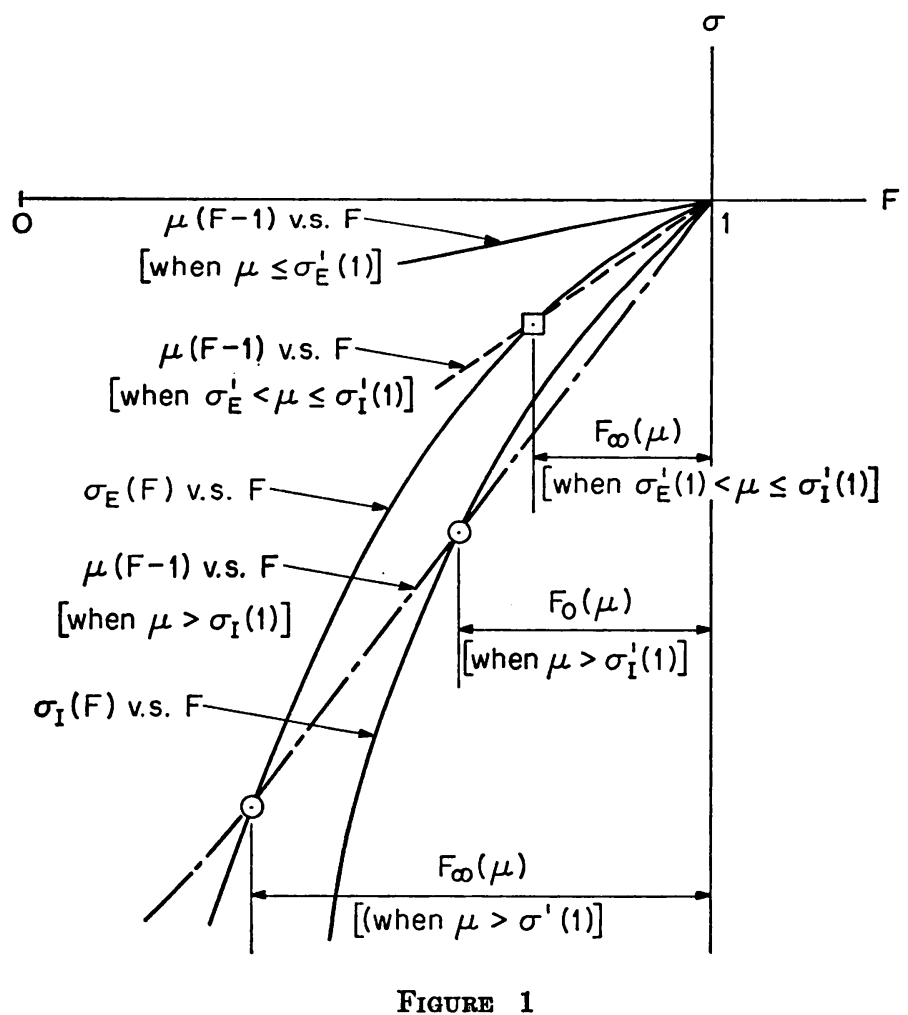


The main results of this investigation are contained in the following theorem:

THEOREM 3.1. (i) If $V_{0} \leq V_{E}$, there is no nontrivial compressive loading solution.

(ii) If $V_{E}<V_{0}<V_{I}$, there exists a nontrivial, $C^{1}$, compressive loading solution. This solution has the property that

$$
\begin{aligned}
& \lim _{\xi \rightarrow \infty} F(\xi)=F_{\infty}, \\
& \lim _{\xi \rightarrow \infty} \sigma(\xi)=\mu\left(F_{\infty}-1\right),
\end{aligned}
$$

where $F_{\infty}$ satisfies (3.10).

(iii) If $V_{I}<V_{0}$, there is no nontrivial, $C^{1}$, compressive loading solution, but there does exist a nontrivial compressive loading solution with the following properties:

(a) The functions $F$ and $\sigma$ are continuous and have continuous first derivatives on $S_{0}$ where

$$
S_{0}=R=\{0\},
$$

(b) $F(\xi) \equiv 1$ and $\sigma(\xi) \equiv 0$ for all $\xi \in(-\infty, 0)$,

(c) $F\left(0^{+}\right)=\lim _{\xi \rightarrow 0^{+}} F(\xi)=F_{0}$ and $\sigma\left(0^{+}\right)=\lim _{\xi \rightarrow 0^{+}} \sigma(\xi)=\mu\left(F_{0}-1\right)$ where $F_{0}$ satisfies (3.12), and

(d) $\lim _{\xi \rightarrow \infty} F(\xi)=F_{\infty}$ and $\lim _{\xi \rightarrow \infty} \sigma(\xi)=\mu\left(F_{\infty}-1\right)$ where $F_{\infty}<F_{0}$ satisfies (3.10).

(iv) If $V_{0}=V_{I}$, there exists a nontrivial compressive loading solution with the following properties:

(a) The function $F$ and $\sigma$ are continuous on $R$ and have continuous first derivatives on $S_{0}$ where $S_{0}$ is defined in (3.16),

(b) $F(\xi)=1$ and $\sigma(\xi)=0$ for all $\xi \in(-\infty, 0)$,

(c)

$$
\lim _{\xi \rightarrow 0^{+}} \frac{d F}{d \xi}=-G_{F}(1,0) \frac{1-E(1,0) / \sigma_{E}^{\prime}(1)}{\left(E_{F}(1,0)+E_{\sigma}(1,0) E(1,0)\right)}<0
$$

and (3.10)

(d) $\lim _{\xi \rightarrow \infty} F(\xi)=F_{\infty}$ and $\lim _{\xi \rightarrow \infty} \sigma(\xi)=E(1,0)\left(F_{\infty}-1\right)$ where again $F_{\infty}$ satisfies

The nontrivial solutions of (ii) are commonly referred to as structured shock waves, those of (iii) as shock waves, and those of (iv) as acceleration waves.

Proof of (i). We assume that assertion (i) is false; i.e. we assume there exists a pair of functions $(F, \sigma) \not \equiv(1,0)$ which are bounded, monotone decreasing, and satisfy (3.1)(3.3). It then follows that there exists a point $\xi_{0} \in R$ such that

$$
F\left(\xi_{0}\right)<1, \quad \sigma\left(\xi_{0}\right)=\mu\left(F\left(\xi_{0}\right)-1\right)<0,
$$

and

$$
\left[\mu-E\left(F\left(\xi_{0}\right), \mu\left(F\left(\xi_{0}\right)-1\right)\right)\right] d F\left(\xi_{0}\right) / d \xi=G\left(F\left(\xi_{0}\right), \mu\left(F\left(\xi_{0}\right)-1\right)\right) .
$$

The inequalities $F\left(\xi_{0}\right)<1$ and $V_{0} \leq V_{E}$ and Eqs. (2.8) and (3.9) $)_{1}$ imply that

$$
E\left(F\left(\xi_{0}\right), \mu\left(F\left(\xi_{0}\right)-1\right)\right)>E(1,0) \geq \mu,
$$




$$
G\left(F\left(\xi_{0}\right), \mu\left(F\left(\xi_{0}\right)-1\right)\right)<0 .
$$

Equations (3.18)-(3.20) then yield

$$
d F\left(\xi_{0}\right) / d \xi>0
$$

which contradicts the assumption that $F$ is compressive loading.

Proof of (ii). It suffices to show that for $V_{E}<V_{0}<V_{I}$ there exists a $C^{1}(-\infty, \infty)$, monotone decreasing function $F$ which satisfies

$$
\begin{gathered}
{[\mu-E(F, \mu(F-1))] \frac{d F}{d \xi}=G(F, \mu(F-1)), \quad \xi \in(-\infty, \infty),} \\
\lim _{\xi \rightarrow-\infty} F(\xi)=1, \\
\lim _{\xi \rightarrow \infty} F(\xi)=F_{\infty}
\end{gathered}
$$

where $F_{\infty}$ is defined by (3.10).

$V_{0}<V_{I}$ and Eq. (2.8a) imply that

$$
\begin{aligned}
-\infty<\mu-E\left(F_{\infty}, \mu\left(F_{\infty}-1\right)\right) & \leq \mu-E(F, \mu(F-1)) \\
& \leq \mu-E(1,0)<0, \quad F \in\left[F_{\infty}, 1\right],
\end{aligned}
$$

and $V_{E}<V_{0}$ and Eqs. (2.8b), (2.9b), (3.2), and (3.10) imply that

$$
G(F, \mu(F-1)) \geq 0, \quad F \in\left[F_{\infty}, 1\right],
$$

with equality holding only if $F=F_{\infty}$ or $F=1$. It now follows from (3.25) and (3.26) and from the smoothness of $E$ and $G$ that for any $\delta \in\left(F_{\infty}, 1\right)$ and $\xi_{0} \in(-\infty, \infty)$ there exists a unique function $F\left(\cdot ; \xi_{0}, \delta\right)$ which is $C^{1}(-\infty, \infty)$, satisfies (3.22), and is such that $F\left(\xi_{0} ; \xi_{0}, \delta\right)=\delta$. Moreover, (3.25) and (3.26) imply that $F\left(\cdot ; \xi_{0}, \delta\right)$ is monotone decreasing and satisfies (3.23) and (3.24), and therefore the proof of (ii) is complete.

Proof of (iii). The proof that there is no nontrivial, $C^{1}(-\infty, \infty)$, compressive loading solution is analogous to the proof of assertion (i).

We now prove the second part of (iii). Since the pair of functions $(F(\xi), \sigma(\xi))=$ $(1,0), \xi \in(-\infty, 0)$, meets (3.1) and satisfies (3.2) and (3.3) in $(-\infty, 0)$, it suffices to show that the function $F(\xi)=1, \xi \in(-\infty, 0)$, has an extension to $(-\infty, \infty)$ with the following properties:

(a) The restriction of $F$ to $(0, \infty)$ is $C^{1}$ and monotone decreasing,

(b) $F$ satisfies $(3.22)$ on $(0, \infty)$, and

(c) $F\left(0^{+}\right)=F_{0}, \lim _{\xi \rightarrow \infty} F(\xi)=F_{\infty}$.

$V_{I}<V_{0}$ and Eqs. (3.13) and (2.8a) imply that

$$
\begin{aligned}
-\infty<\mu-E\left(F_{\infty}, \mu\left(F_{\infty}-1\right)\right) & \leq \mu-E(F, \mu(F-1)) \\
& \leq \mu-E\left(F_{0}, \mu\left(F_{0}-1\right)\right)<0, \quad F \in\left[F_{\infty}, F_{0}\right],
\end{aligned}
$$

and $V_{E}<V_{0}$ and Eqs. (2.8b), (2.9b), (3.2), and (3.10) imply that

$$
G(F, \mu(F-1)) \geq 0, \quad F \in\left[F_{\infty}, F_{0}\right]
$$


with equality holding only if $F=F_{\infty}$. Equations (3.27) and (3.28) and the smoothness of $E$ and $G$ guarantee the existence of unique function $F\left(\cdot ; 0^{+}, F_{0}\right)$ which is $C^{1}(0, \infty)$, satisfies $(3.22)$ on $(0, \infty)$, and is such that $\lim _{\xi \rightarrow 0^{+}} F\left(\xi ; 0^{+}, F_{0}\right)=F_{0}$. Moreover, (3.27) and (3.28) imply that $F\left(\cdot ; 0^{+}, F_{0}\right)$ is monotone decreasing on $(0, \infty)$ and that $\lim _{\xi \rightarrow \infty} F(\xi$; $\left.0^{+}, F_{0}\right)=F_{\infty}$. Using $F\left(\cdot ; 0^{+}, F_{0}\right)$ we may now extend the function $F(\xi) \equiv 1, \xi \in(-\infty, 0)$, to $(-\infty, \infty)$ in such a way that (a), (b), and (c) hold and therefore the proof of (iii) is complete.

Proof of (iv). Since the pair of functions $(F(\xi), \sigma(\xi))=(1,0), \xi \in(-\infty, 0)$, meets (3.1) and satisfies (3.2) and (3.3) in $(-\infty, 0)$, it suffices to show that the function $F(\xi)=$ $1, \xi \in(-\infty, 0)$, has an extension to $(-\infty, \infty)$ with the following properties:

(a) the restriction of $F$ to $(0, \infty)$ is $C^{1}$ and monotone decreasing,

(b) $F$ satisfies $(3.22)$ on $(0, \infty)$, and

(c)

$$
F\left(0^{+}\right)=1, \quad \lim _{\xi \rightarrow 0^{+}} \frac{d F}{d \xi}=-\frac{\left(G_{F}(1,0)+G_{\sigma}(1,0) E(1,0)\right)}{\left(E_{F}(1,0)+E_{\sigma}(1,0) E(1,0)\right)},
$$

and $\lim _{\xi \rightarrow \infty} F(\xi)=F_{\infty}$.

We define the function $\mathfrak{H}:(0,1] \rightarrow(-\infty, 0]$ as follows:

$$
\begin{gathered}
\mathcal{H}(F)=\frac{G(F, E(1,0)(F-1))}{E(1,0)-E(F, E(1,0)(F-1))}, \quad F \in(0,1), \\
\mathcal{H}(1)=-\frac{\left(G_{F}(1,0)+G_{\sigma}(1,0) E(1,0)\right)}{\left(E_{F}(1,0)+E_{\sigma}(1,0) E(1,0)\right)}<0,
\end{gathered}
$$

and note that part (a) of Assumption 2.1 implies that

(i) $\operatorname{TC}$ is continuous on $\left[F_{\infty}, 1\right]$, and

(ii) $\operatorname{HC}(F) \leq 0$ for all $F \in\left[F_{\infty}, 1\right]$ with equality holding only if $F=F_{\infty}$.

It now follows from standard theorems in ordinary differential equations that there exists at least one $C^{1}[0, \infty)$, monotone decreasing function $F\left(\cdot ; 0^{+}, 1\right)$ satisfying

$$
\begin{gathered}
\frac{d F}{d \xi}=\mathfrak{H C}\left(F^{\prime}\right), \quad \xi \geq 0, \\
F\left(0^{+} ; 0^{+}, 1\right)=1
\end{gathered}
$$

and having the following additional properties:

$$
\begin{gathered}
\lim _{\xi \rightarrow 0^{+}} \frac{d F(\xi)}{d \xi}=-\frac{\left(G_{F}(1,0)+G_{\sigma}(1,0) E(1,0)\right)}{\left(E_{F}(1,0)+E_{\sigma}(1,0) E(1,0)\right)}, \\
\lim _{\xi \rightarrow \infty} F\left(\xi ; 0^{+}, 1\right)=F_{\infty} .
\end{gathered}
$$

Using $F\left(\cdot ; 0^{+}, 1\right)$ we may extend the function $F(\xi)=1, \xi \in(-\infty, 0)$, to $(-\infty, \infty)$ in such a way that (a), (b), and (c) hold, and therefore the proof of (iv) is complete.

Acknowledgements. The author wishes to thank Dr. Bernard D. Coleman and Professor Morton E. Gurtin for valuable discussions.

This research was supported by the Air Force Office of Scientific Research under Grant No. AF-AFOSR 728-66. 


\section{REFERENCES}

1. B. D. Coleman, M. E. Gurtin, and I. Herrera R., Arch. Rational Mech. Anal. 19, 1-18 (1965)

2. B. D. Coleman and M. E. Gurtin, Arch. Rational Mech. Anal. 19, 239-265 (1965)

3. B. D. Coleman and M. E. Gurtin, Arch. Rational Mech. Anal. 19, 266-298 (1965)

4. B. D. Coleman and M. E. Gurtin, Arch. Rational Mech. Anal. 19, 317-338 (1965)

5. E. Varley, Arch. Rational Mech. Anal. 19, 215-225 (1965)

6. B. D. Coleman, J. M. Greenberg and M. E. Gurtin, Arch. Rational Mech. Anal. 22, 333-354 (1966)

7. C. -C. Wang and R. M. Bowen, Arch. Rational Mech. Anal. 22, 79-99 (1966)

8. J. Dunwoody, Intl. J. Engng. Sci. 4, 277-287, (1966)

9. A. C. Pipkin, Quart. Appl. Math. XXIII, No. 4, 297-303 (1966)

10. J. M. Greenberg, Arch. Rational Mech. Anal. 24, 1-21 (1967)

11. W. Noll, J. Rational Mech. Anal. 4, 3-81 (1955) 